\title{
PLANT GROWTH REGULATORS AND SIMULATED HAIL- DAMAGE IMPROVE ROSE-GERANIUM (Pelargonium graveolens L'Hér.) LEAF MINERAL STATUS AND PHENOLIC COMPOSITION
}

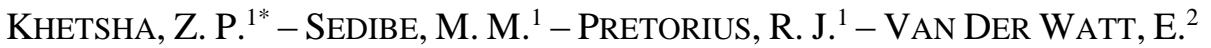 \\ ${ }^{1}$ Central University of Technology, Free State, Department of Agriculture, Private Bag X \\ 20539, Bloemfontein 9301, South Africa \\ ${ }^{2}$ University of the Free State, Department of Soil, Crop and Climate Sciences, Post Office Box \\ 339, Bloemfontein 9300, South Africa \\ ${ }^{*}$ Corresponding author \\ e-mail: zkhetsha@cut.ac.za; phone:+27-51-507-3130 \\ (Received $8^{\text {th }}$ Feb 2021; accepted $14^{\text {th }}$ May 2021)
}

\begin{abstract}
This study aimed to evaluate the effects of plant growth regulators (PGRs) on the leaf mineral status, and the phenolic compounds of rose geranium with stimulated hail damage. This experiment was conducted in a $3 \times 4$ factorial treatment design laid out in a randomized complete block design. The interaction between simulated hail damage and PGRs significantly affected the C, N, P, and Ca content, as well as the $\mathrm{C}: \mathrm{N}$ ratio. A principal component analysis was performed to increase the interpretability of leaf mineral data. PC1 and PC2 accounted for $73.01 \%$ of the total variance, confirming that hail damaged rose geranium plants accumulate $\mathrm{N}, \mathrm{P}, \mathrm{K}, \mathrm{Mg}, \mathrm{Ca}, \mathrm{Mn}$, and $\mathrm{Na}$ when treated with high levels of PGRs (gibberellic acid- $2.55 \mathrm{mg} / \mathrm{kg}$, brassinosteroids $-1.02 \mathrm{mg} / \mathrm{kg}$, and cytokinins- $0.05 \mathrm{mg} / \mathrm{kg}$, and the gibberellic acid-3.83 mg/kg, brassinosteroids- $1.53 \mathrm{mg} / \mathrm{kg}$, and cytokinins $-0.075 \mathrm{mg} / \mathrm{kg}$ ). Citric acid, coumaroyl tyrosine, 5-Heptadecylresorcinol and kaempferol-O-hexoside-O-deoxy-hexose were among the phenolic compounds affected by simulated hail damage. PC1 and PC2 accounted for most variability (41.30\%) of the phenolic compounds, confirming that severely hail-damaged plants (100\%) accumulate most of the phenolics. Post-hail damage recovery strategies using PGRs may be an alternative strategy for improving leaf mineral status, assisting the plants to recover lost herbage material.
\end{abstract}

Keywords: brassinosteroids, cytokinin, defoliation, gibberellic acid, carbon to nitrogen ratio

\section{Introduction}

Abiotic stresses affect plant growth and development at the physiological, biochemical, and molecular levels, which can lead to a reduction in crop productivity (Wani et al., 2016). Amongst the abiotic stress conditions, hail damage is one of the most severe environmental factors, causing defoliation and wounded stems and flowers (Wani et al., 2016). Hailstones may cause significant damage to aromatic and medicinal plants such as rose geranium (Pelargonium graveolens L'Hér.). Following hail damage, plants suffer a metabolic shock, directly affecting the physiological processes such as the photosynthesis rate, the nutrient balance, and accumulation of the phenolic compounds through the phenylpropanoids metabolism (Pankoke and Müller, 2013; Smékalová et al., 2014).

Phenolics provide cytotoxic effects, as well as the building blocks for polyphenolicbased cell wall modifications which assist with organ regeneration in defoliated or wounded plants (Nanda and Melnyk, 2018; Kim et al., 2019). The level of endogenous phenolics in refoliating plants influences plant growth and development, clearly indicating the relation of the leaf refoliation with the leaf ontogeny (Maia et al., 2007). 
The accumulation of the plant phenolics may be associated with the mineral balance in the plant. According to Treutter (2010), maintenance of minerals is a prerequisite for providing co-factors for many enzymes of the phenolic pathway.

The complexity of the total minerals and phenolic pathways from the treated plants may result in substantial and complex data (Mishra et al., 2018). Multivariate data analysis is a suitable technique to reduce the dimensionality of such datasets, increasing interpretability but at same time minimizing information loss.

Aspects relating to changes in mineral acquisition, photosynthetic activities, and the phenolics are primarily driven by upregulation of endogenous phytohormones (Ikeuchi et al., 2017; Nanda and Melnyk, 2018). According to Parađiković et al. (2019), exogenous application of agricultural plant growth regulators (PGRs) may effectively improve nutrient acquisition, and increases the antioxidant capacity of plant tissue, directly stimulating the vegetative growth. Therefore, post-hail damage recovery method using PGRs may be an alternative strategy for improving the leaf mineral status and the phenolic composition, assisting the plants to recover lost herbage material. This study aimed to determine the ability of exogenous PGRs application to improve the leaf mineral status and the phenolic compounds of a hail-damaged rose geranium.

\section{Materials and methods}

\section{Study site and general crop management practices}

This experiment was conducted in a $72 \mathrm{~m}^{2}$ temperature-controlled, plastic-covered greenhouse for 122 days using a drain-to-waste hydroponic system, on the campus of the Central University of Technology, Free State. The temperature in the greenhouse was maintained by a fan, which was triggered at ca. $26^{\circ} \mathrm{C}$.

Rooted rose geranium cuttings 'Bourbon-type' (ca. $10 \mathrm{~cm}$ tall) were sourced and obtained from a reputable commercial grower (Siyakholwa Development Foundation, RSA). These rooted cuttings were transplanted into $5 \mathrm{~L}$ potting bags filled with a sterile silica-sand root-medium, with a standard average grain size diameter of $2 \mathrm{~mm}$. A single rooted cutting was transplanted into each potting container.

Water analysis was conducted during both growing seasons and taken into account during the formulation of the nutrient solution. Plants were fertigated three times per day (8:00 am, 12:00 pm, and 4:00 pm) using a 'drain to waste' drip irrigation system, where each dripper supplied $2 \mathrm{~L} / \mathrm{h}$ of water and nutrient solution (Sedibe and Allemann, 2012). Initially, fertigation intervals for the young plants lasted for five min, containing a balanced nutrient solution, as recommended for rose geranium (Sedibe and Allemann, 2012). As the plants matured and the demand for water and nutrients increased, the applied volumes were increased to ensure that ca. $10 \%$ to $25 \%$ of the solution drained to waste, in order to prevent salt accumulation in the growing media (Combrink, 2005; Sedibe and Allemann, 2012). The electric conductivity (EC) and $\mathrm{pH}$ of the nutrient solutions were maintained at $1.6 \mathrm{mS} / \mathrm{cm}$ and 5.5, respectively. Using a $\mathrm{pH}$ and EC meter (Hanna HI 98129 Digital meter), the desired pH and EC levels were achieved by using nitric acid and adjusting the nutrient solution concentration to reach the desired EC (Combrink, 2005). No phytophagous pests were documented during the experiment, however, Malasol (an organophosphate) was preventatively sprayed at $1.75 \mathrm{ml} / \mathrm{L}$ throughout the cropping seasons. These applications were repeated for three to six days, at four-week intervals. 


\section{Treatments}

A 3 x 4 factorial design, arranged in a randomized complete block design was used, and had three replications. Treatments consisted of three hail damage simulation levels (0\% [intact plants], $50 \%$, and $100 \%$ leaf defoliation). The levels of PGRs-mixtures evaluated for the level 1 had gibberellic acid (GA)-1.26 mg/kg, brassinosteroids (BR)-0.51 mg/kg, and traces of cytokinins (CKs)-0.025 mg/kg; level 2 had GA-2.55 mg/kg, BR-1.02 mg/kg, and traces of CKs-0.05 mg/kg; level 3 had GA-3.83 mg/kg, BR-1.53 mg/kg, and traces of CKs-0.075 mg/kg, and the control (consisting of distilled water, zero\% $\mathrm{GA}$, zero\% $\mathrm{BR}$, and zero\% $\mathrm{CKs}$ ).

Hail damage was simulated 61 after transplanting days using manually-operated garden secateurs, following the procedures described by Irigoyen et al. (2010) and Nielsen (2012) at ca. 14:00. The 50\% hail damage simulation level was achieved by defoliating the top half (average plant height per experimental unit) of each experimental plant (Obeso, 2002). The 100\% hail damage simulation treatment was achieved by the total removal of all leaves (Changnon, 1999; Obeso, 2002). This was followed by the removal of stem terminal buds and random bruising of the main stem and lateral branches (using a pair of hand secateurs) on the same day. Plants were subsequently sprayed with a fine water mist, using a nozzle calibrated precision sprayer (0.3 $\mathrm{MPa}$ pressure), to simulate humid summer afternoon conditions (Irigoyen et al., 2010; Bal et al., 2014). Subsequently, PGRs application treatments followed same on the day, an hour after simulated hail damage. A full cover spray of approximately $50 \mathrm{~mL}$ of PGRs treatment solution was applied to each plant. Treatments were subsequently repeated every 14 days between 13:00 and 14:00, and this continued until harvesting that occurred on 31 December 2019 (Table 1).

Table 1. Time chart for the application from transplant to harvest (2019), including treatments

\begin{tabular}{c|c|c|c|c|c|c|c}
\hline 01-Sep & 31-Oct & 07-Nov & 14-Nov & 21-Nov & 28-Nov & 05-Dec & 12-Dec \\
\hline Transplanting & HD + PGRs $1^{\text {st }}$ & No appl. & PGRs $2^{\text {nd }}$ & No appl. & PGRs $3^{\text {rd }}$ & No appl. & PGRs $4^{\text {th }} \&$ Harvest \\
\hline
\end{tabular}

$\mathrm{HD}=$ Hail damage simulation; No appl. = No application

\section{Parameters}

Leaf minerals ( $\mathrm{P}, \mathrm{K}, \mathrm{Ca}, \mathrm{Mg}, \mathrm{B}, \mathrm{Mn}, \mathrm{Zn}, \mathrm{Fe}, \mathrm{Cu}$ and $\mathrm{Na}$ ) were analyzed using an inductively coupled plasma Optical Emission Spectroscopy instrument (Thermo iCAP 6000 series, Thermo Scientific, USA) (Masson et al., 2010). Carbon, N and S were analyzed using Vario EL Cube Elemental analyzer (Elementar Vario PYRO cube, Elementary, Hanau, Germany) (Dhaliwal et al., 2014).

For the phenolic profile assays, the plant leaf material was oven-dried (ECOt herm labotec oven, RSA) at a temperature of $60^{\circ} \mathrm{C}$, for a duration of 48 hours. Approximately $2 \mathrm{~g}$ of dry leaves were ground in a micro hammer mill (Culatti, Zurich). Fine milled leaf material was then extracted with $50 \%$ methanol in water containing $1 \%$ formic acid $(15 \mathrm{~mL})$ in a $50 \mathrm{~mL}$ polypropylene centrifuge tube by soaking it overnight. This was followed by extraction in an ultrasonic bath $(0.5 \mathrm{~Hz}$, Integral Systems, RSA) for $60 \mathrm{~min}$ at room temperature. The extracts were centrifuged (Hermle Z160m, $3000 \mathrm{~g}$ for $5 \mathrm{~min}$ ) and transferred to vials. Stock solutions were prepared quantitatively in cocktails ranging from 1 to $100 \mu \mathrm{g} / \mathrm{ml}$ in concentration. The solvent used for the preparation of the cocktails 
was $50 \%$ methanol in water, containing $1 \%$ formic acid, which was the same as that used for extraction of the leaf samples.

A Waters Synapt G2 Quadrupole time-of-flight (QTOF) mass spectrometer (MS) connected to a Waters Acquity ultra-performance liquid chromatography (UPLC) (Waters, Milford, MA, USA) was used for analysis. Electrospray ionization was used in negative mode with a cone voltage of $15 \mathrm{~V}$, and a desolvation temperature of $275^{\circ} \mathrm{C}$, desolvation gas at $650 \mathrm{~L} / \mathrm{h}$. The rest of the MS settings optimized for best resolution and sensitivity. Data were acquired by scanning from 150 to $1500 \mathrm{~m} / \mathrm{z}$ in resolution mode, as well as in MSE mode. In MSE mode, two channels of MS data were acquired, one at low collision energy (4 V) and the second using a collision energy ramp (40-100 V) also to obtain fragmentation data. Leucine enkaphalin was used as lock mass (reference mass) for accurate mass determination, and the instrument was calibrated with sodium formate. The separation was achieved on a Waters HSS T3, $2.1 \times 100 \mathrm{~mm}, 1.7 \mu \mathrm{m}$ column. An injection volume of $2 \mu \mathrm{L}$ was used, and the mobile phase consisted of $0.1 \%$ formic acid (solvent A) and acetonitrile containing $0.1 \%$ formic acid as solvent B. The gradient started at $100 \%$ with solvent $\mathrm{A}$ for $1 \mathrm{~min}$ and, then changed to $28 \%$ solvent $\mathrm{B}$ for $22 \mathrm{~min}$ in a linearly. The gradient further went to $40 \% \mathrm{~B}$ over $50 \mathrm{~s}$ and a wash step of $1.5 \mathrm{~min}$ at $100 \%$ $\mathrm{B}$, followed by re-equilibration to initial conditions for $4 \mathrm{~min}$. The flow rate was $0.3 \mathrm{ml} / \mathrm{min}$, and the column temperature was maintained at $55^{\circ} \mathrm{C}$. Ion mobility data were obtained using the same UPLC gradient and column as above, and IMS Wave velocity was set at $332 \mathrm{~m} / \mathrm{s}$, and wave height at $20.2 \mathrm{~V}$. Polyalanine was used for the calibration and calculations (Stander et al., 2017).

\section{Statistical analysis}

All parameters were statistically analyzed and compared using PROC GLIMMIX, SAS version 9.4 (PROC GLIMMIX, SAS Institute 2013). Significantly different means among the treatments were separated using Tukey's least significant difference ad hoc mean comparison tests, at the 0.05 level of significance (Steel and Tourie, 1980). The Shapiro-Wilks test was performed on standardized residuals to test for any deviations from normality (Shapiro and Wilk, 1965). Multivariate data analysis, using a principal component analysis (PCA; PCA-XLSTAT 2015), was then used to identify and evaluate the groupings between the variables.

\section{Results}

\section{Leaf minerals}

The concentrations of $\mathrm{K}, \mathrm{Mg}, \mathrm{S}, \mathrm{B}, \mathrm{Cu}, \mathrm{Fe}, \mathrm{Mn}$, and $\mathrm{Zn}$ were affected by the simulated hail damage, but responses differed between the PGR levels (Table 2). K and Zn content was significantly higher $(P<0.05)$ in plants subjected to simulated hail damage (at any level), indicating that refoliating plants accumulate $\mathrm{K}$ and $\mathrm{Zn}$. The highest $\mathrm{Mg}, \mathrm{Cu}$ and Mn content, of $6.82 \mathrm{mg} / \mathrm{kg}, 7.83 \mu \mathrm{g} / \mathrm{kg}$, and $236.73 \mu \mathrm{g} / \mathrm{kg}$, respectively, was measured in plants subjected to $100 \%$ simulated hail damage. The content of S, B and Fe declined significantly $(P<0.05)$ in $100 \%$ simulated hail-damaged plants, compared with the $50 \%$ simulated hail-damaged and intact plants. 
Table 2. The effects of hail-simulated damage (HD) and the application of simulated hail-damaged (PGRs) on leaf minerals of rose geranium plant leaves

\begin{tabular}{|c|c|c|c|c|c|c|c|c|c|c|c|c|c|c|}
\hline \multirow[b]{2}{*}{ Treatments } & \multicolumn{14}{|c|}{ Mineral Composition } \\
\hline & $\begin{array}{c}\mathrm{C} \\
\mathrm{mg} / \mathrm{kg}\end{array}$ & $\begin{array}{c}\mathrm{N} \\
\mathrm{mg} / \mathrm{kg}\end{array}$ & $\mathrm{C}: \mathrm{N}$ & $\begin{array}{c}\mathrm{P} \\
\mathrm{mg} / \mathrm{kg}\end{array}$ & $\begin{array}{c}\mathrm{K} \\
\mathrm{mg} / \mathrm{kg}\end{array}$ & $\begin{array}{c}\mathrm{Ca} \\
\mathrm{mg} / \mathrm{kg}\end{array}$ & $\begin{array}{c}\mathrm{Mg} \\
\mathrm{mg} / \mathrm{kg}\end{array}$ & $\begin{array}{c}\mathrm{S} \\
\mathrm{mg} / \mathrm{kg}\end{array}$ & $\begin{array}{c}\text { B } \\
\mathrm{ug} / \mathrm{kg}\end{array}$ & $\begin{array}{c}\mathrm{Cu} \\
\mathrm{ug} / \mathrm{kg}\end{array}$ & $\begin{array}{c}\mathrm{Fe} \\
\mathrm{mg} / \mathrm{kg}\end{array}$ & $\begin{array}{c}\text { Mn } \\
\text { ug/kg }\end{array}$ & $\begin{array}{c}\mathrm{Zn} \\
\mathrm{ug} / \mathrm{kg}\end{array}$ & $\begin{array}{c}\mathrm{Na} \\
\mathrm{mg} / \mathrm{kg}\end{array}$ \\
\hline Hail damage & & & & & & & & & & & & & & \\
\hline $0 \%$ defoliation & $44.41^{\mathrm{a}}$ & $3.57^{\mathrm{b}}$ & $12.45^{\mathrm{a}}$ & $3.78^{\mathrm{b}}$ & $30.52^{\mathrm{b}}$ & $16.21^{\mathrm{a}}$ & $3.63^{\mathrm{b}}$ & $50.54^{\mathrm{a}}$ & $130.15^{\mathrm{a}}$ & $7.11^{\mathrm{b}}$ & $0.97^{\mathrm{a}}$ & $89.71^{\mathrm{c}}$ & $61.57^{\mathrm{b}}$ & $2.17^{\mathrm{a}}$ \\
\hline $50 \%$ defoliation & $43.56^{\mathrm{ab}}$ & $3.75^{\mathrm{b}}$ & $11.72^{\mathrm{a}}$ & $3.93^{\mathrm{ab}}$ & $39.90^{\mathrm{a}}$ & $14.08^{\mathrm{b}}$ & $3.87^{\mathrm{b}}$ & $45.93^{\mathrm{a}}$ & $129.57^{\mathrm{a}}$ & $7.18^{\mathrm{b}}$ & $0.93^{\mathrm{a}}$ & $132.70^{\mathrm{b}}$ & $79.99^{\mathrm{a}}$ & $1.89^{\mathrm{a}}$ \\
\hline $100 \%$ defoliation & $41.95^{\mathrm{b}}$ & $3.97^{\mathrm{a}}$ & $10.64^{\mathrm{b}}$ & $4.37^{\mathrm{a}}$ & $44.59^{\mathrm{a}}$ & $11.81^{\mathrm{c}}$ & $6.82^{\mathrm{a}}$ & $33.30^{\mathrm{b}}$ & $109.86^{\mathrm{b}}$ & $7.83^{\mathrm{a}}$ & $0.33^{\mathrm{b}}$ & $236.73^{\mathrm{a}}$ & $80.36^{\mathrm{a}}$ & $1.81^{\mathrm{a}}$ \\
\hline $\mathrm{LSD}_{\mathrm{T} 0.05}$ & $2.28 *$ & $0.16^{*}$ & $0.77 *$ & $0.59 *$ & $7.76^{*}$ & $1.84 *$ & $0.76^{*}$ & $7.73 *$ & $11.42 *$ & $0.41 *$ & $0.41 *$ & $35.09 *$ & $12.15^{*}$ & $0.46^{\mathrm{ns}}$ \\
\hline PGRs & & & & & & & & & & & & & & \\
\hline & $44.35^{\mathrm{a}}$ & $3.70^{\mathrm{a}}$ & $11.74^{\mathrm{a}}$ & $4.17^{\mathrm{a}}$ & $29.09^{b}$ & $9.51^{\mathrm{c}}$ & $3.84^{\mathrm{b}}$ & $47.76^{\mathrm{a}}$ & $129.65^{\mathrm{a}}$ & $7.62^{\mathrm{a}}$ & $0.74^{\mathrm{a}}$ & $158.73^{\mathrm{a}}$ & $75.34^{\mathrm{a}}$ & $2.19^{\mathrm{a}}$ \\
\hline Level 1 & $43.73^{\mathrm{a}}$ & $3.74^{\mathrm{a}}$ & $11.36^{\mathrm{a}}$ & $4.06^{\mathrm{a}}$ & $39.47^{\mathrm{a}}$ & $12.27^{\mathrm{b}}$ & $5.10^{\mathrm{a}}$ & $42.18^{\mathrm{ab}}$ & $124.57^{a}$ & $7.58^{\mathrm{a}}$ & $0.75^{\mathrm{a}}$ & $142.11^{\mathrm{a}}$ & $79.89^{\mathrm{a}}$ & $2.08^{\mathrm{a}}$ \\
\hline Level 2 & $43.36^{\mathrm{a}}$ & $3.79^{\mathrm{a}}$ & $11.44^{\mathrm{a}}$ & $4.02^{\mathrm{a}}$ & $42.46^{\mathrm{a}}$ & $17.28^{\mathrm{a}}$ & $4.54^{\mathrm{ab}}$ & $45.96^{\mathrm{ab}}$ & $123.27^{\mathrm{a}}$ & $7.61^{\mathrm{a}}$ & $0.81^{\mathrm{a}}$ & $133.75^{\mathrm{a}}$ & $71.40^{\mathrm{a}}$ & $1.88^{\mathrm{a}}$ \\
\hline Level 3 & $41.79^{\mathrm{a}}$ & $3.76^{\mathrm{a}}$ & $11.87^{\mathrm{a}}$ & $3.82^{\mathrm{a}}$ & $43.17^{\mathrm{a}}$ & $17.14^{\mathrm{a}}$ & $5.11^{\mathrm{a}}$ & $37.14^{\mathrm{b}}$ & $115.29^{a}$ & $6.68^{\mathrm{b}}$ & $0.59^{\mathrm{a}}$ & $154.71^{\mathrm{a}}$ & $68.32^{\mathrm{a}}$ & $1.68^{\mathrm{a}}$ \\
\hline LSD $_{\mathrm{T} 0.05}$ & $3.64^{\mathrm{ns}}$ & $0.18^{\mathrm{ns}}$ & $0.89^{\text {ns }}$ & $0.68^{\mathrm{ns}}$ & $9.22 *$ & $2.13^{*}$ & $0.88^{*}$ & $8.93 *$ & $15.19^{\mathrm{ns}}$ & $0.01 *$ & $0.47^{\mathrm{ns}}$ & $40.61^{\mathrm{ns}}$ & $14.04^{\mathrm{ns}}$ & $0.53^{\mathrm{ns}}$ \\
\hline HD x PGRs & & & & & & & & & & & & & & \\
\hline $0 \% \times$ Control & $45.45^{\mathrm{a}}$ & $3.46^{\mathrm{f}}$ & $13.13^{\mathrm{a}}$ & $4.23^{\mathrm{a}}$ & $39.07^{\mathrm{a}}$ & $14.64^{\mathrm{ed}}$ & $4.25^{\mathrm{a}}$ & $49.93^{\mathrm{a}}$ & $132.19^{a}$ & $8.05^{\mathrm{a}}$ & $0.72^{\mathrm{a}}$ & $95.81^{\mathrm{a}}$ & $60.71^{\mathrm{a}}$ & $2.34^{\mathrm{a}}$ \\
\hline $0 \%$ x Level 1 & $44.29^{\mathrm{a}}$ & $3.62^{\mathrm{def}}$ & $12.23^{\mathrm{a}}$ & $4.07^{\mathrm{ab}}$ & $40.06^{\mathrm{a}}$ & $15.46^{\text {cde }}$ & $3.91^{\mathrm{a}}$ & $49.78^{a}$ & $134.79^{a}$ & $7.42^{\mathrm{a}}$ & $0.89^{\mathrm{a}}$ & $86.89^{a}$ & $62.35^{\mathrm{a}}$ & $2.28^{\mathrm{a}}$ \\
\hline $0 \% \times$ Level 2 & $43.24^{\mathrm{a}}$ & $3.67^{\text {bcdef }}$ & $11.78^{\mathrm{ab}}$ & $3.60^{\mathrm{ab}}$ & $40.11^{\mathrm{a}}$ & $16.33^{\mathrm{bcd}}$ & $3.77^{\mathrm{a}}$ & $47.05^{\mathrm{a}}$ & $135.41^{\mathrm{a}}$ & $6.78^{\mathrm{a}}$ & $0.99^{\mathrm{a}}$ & $95.58^{\mathrm{a}}$ & $65.37^{\mathrm{a}}$ & $2.13^{\mathrm{a}}$ \\
\hline $0 \%$ x Level 3 & $43.67^{\mathrm{a}}$ & $3.53^{\text {ef }}$ & $12.37^{\mathrm{a}}$ & $3.83^{\mathrm{ab}}$ & $40.36^{\mathrm{a}}$ & $18.43^{\mathrm{abc}}$ & $4.56^{\mathrm{a}}$ & $36.97^{\mathrm{a}}$ & $118.21^{\mathrm{a}}$ & $6.18^{\mathrm{a}}$ & $1.27^{\mathrm{a}}$ & $80.58^{\mathrm{a}}$ & $57.85^{\mathrm{a}}$ & $1.91^{\mathrm{a}}$ \\
\hline $50 \% \times$ Control & $44.28^{\mathrm{a}}$ & $3.52^{\mathrm{ef}}$ & $12.57^{\mathrm{a}}$ & $4.55^{\mathrm{a}}$ & $53.95^{\mathrm{a}}$ & $15.30^{\text {cde }}$ & $5.10^{\mathrm{a}}$ & $59.97^{\mathrm{a}}$ & $114.91^{\mathrm{a}}$ & $7.21^{\mathrm{a}}$ & $1.03^{\mathrm{a}}$ & $154.92^{\mathrm{a}}$ & $89.54^{\mathrm{a}}$ & $2.22^{\mathrm{a}}$ \\
\hline $50 \%$ x Level 1 & $43.19^{\mathrm{a}}$ & $3.80^{\text {bcde }}$ & $11.86^{\mathrm{abc}}$ & $3.87^{\mathrm{ab}}$ & $41.76^{\mathrm{a}}$ & $19.32^{\mathrm{ab}}$ & $4.37^{\mathrm{a}}$ & $52.49^{\mathrm{a}}$ & $125.19^{a}$ & $7.41^{\mathrm{a}}$ & $0.92^{\mathrm{a}}$ & $141.74^{\mathrm{a}}$ & $84.68^{\mathrm{a}}$ & $2.14^{\mathrm{a}}$ \\
\hline $50 \%$ x Level 2 & $43.52^{\mathrm{a}}$ & $3.59^{\mathrm{ef}}$ & $12.13^{\mathrm{ab}}$ & $3.82^{\mathrm{ab}}$ & $41.63^{\mathrm{a}}$ & $20.81^{\mathrm{a}}$ & $4.23^{\mathrm{a}}$ & $47.00^{\mathrm{a}}$ & $132.44^{\mathrm{a}}$ & $7.95^{\mathrm{a}}$ & $0.71^{\mathrm{a}}$ & $133.16^{\mathrm{a}}$ & $77.27^{\mathrm{a}}$ & $2.42^{\mathrm{a}}$ \\
\hline $50 \%$ x Level 3 & $43.25^{\mathrm{a}}$ & $4.02^{\mathrm{ab}}$ & $10.77^{\mathrm{bcd}}$ & $3.05^{\mathrm{ab}}$ & $41.33^{\mathrm{a}}$ & $20.92^{\mathrm{a}}$ & $4.81^{\mathrm{a}}$ & $42.71^{\mathrm{a}}$ & $145.76^{\mathrm{a}}$ & $6.18^{\mathrm{a}}$ & $1.14^{\mathrm{a}}$ & $100.99^{\mathrm{a}}$ & $71.38^{\mathrm{a}}$ & $2.78^{\mathrm{a}}$ \\
\hline $100 \%$ x Control & $43.65^{\mathrm{a}}$ & $3.76^{\text {bcdef }}$ & $11.67^{\mathrm{abc}}$ & $2.85^{\mathrm{b}}$ & $43.21^{\mathrm{a}}$ & $14.19^{\text {de }}$ & $4.70^{\mathrm{a}}$ & $27.45^{\mathrm{a}}$ & $105.18^{a}$ & $7.59^{\mathrm{a}}$ & $0.24^{\mathrm{a}}$ & $184.08^{\mathrm{a}}$ & $88.24^{\mathrm{a}}$ & $1.07^{\mathrm{a}}$ \\
\hline 100\% x Level 1 & $38.51^{\mathrm{b}}$ & $3.92^{\mathrm{abcd}}$ & $9.70^{\mathrm{d}}$ & $4.17^{\mathrm{a}}$ & $45.71^{\mathrm{a}}$ & $14.10^{\text {de }}$ & $5.01^{\mathrm{a}}$ & $34.05^{\mathrm{a}}$ & $108.39^{a}$ & $7.67^{\mathrm{a}}$ & $0.30^{\mathrm{a}}$ & $238.89^{\mathrm{a}}$ & $82.18^{\mathrm{a}}$ & $1.91^{\mathrm{a}}$ \\
\hline 100\% x Level 2 & $42.32^{\mathrm{a}}$ & $3.96^{\mathrm{abc}}$ & $10.68^{\mathrm{cd}}$ & $4.41^{\mathrm{a}}$ & $48.16^{\mathrm{a}}$ & $14.17^{\text {de }}$ & $5.15^{\mathrm{a}}$ & $34.05^{\mathrm{a}}$ & $113.11^{\mathrm{a}}$ & $8.03^{\mathrm{a}}$ & $0.35^{\mathrm{a}}$ & $244.08^{\mathrm{a}}$ & $78.17^{\mathrm{a}}$ & $2.16^{\mathrm{a}}$ \\
\hline $100 \% \times$ Level 3 & $43.32^{\mathrm{a}}$ & $4.16^{\mathrm{a}}$ & $10.41^{\mathrm{bcd}}$ & $4.51^{\mathrm{a}}$ & $48.03^{\mathrm{a}}$ & $12.26^{\mathrm{e}}$ & $5.13^{\mathrm{a}}$ & $35.46^{\mathrm{a}}$ & $112.75^{\mathrm{a}}$ & $8.03^{\mathrm{a}}$ & $0.41^{\mathrm{a}}$ & $244.76^{\mathrm{a}}$ & $71.38^{\mathrm{a}}$ & $2.13^{\mathrm{a}}$ \\
\hline $\mathrm{LSD}_{\mathrm{T} 0.05}$ & $4.57 *$ & $0.32 *$ & $1.54 *$ & $1.29 *$ & $16.84^{\mathrm{ns}}$ & $3.71 *$ & $2.87^{\text {ns }}$ & $33.47^{\mathrm{ns}}$ & $42.85^{\mathrm{ns}}$ & $1.82^{\text {ns }}$ & $1.87^{\mathrm{ns}}$ & $173.39^{\text {ns }}$ & $34.44^{\mathrm{ns}}$ & $1.92^{\mathrm{ns}}$ \\
\hline
\end{tabular}

The same superscript letter within a column denotes non significance $(\mathrm{P}<0.05) ; \mathrm{ns}=$ not significant; $*$ significant at $\mathrm{P}<0.05$

APPLIED ECOLOGY AND ENVIRONMENTAL RESEARCH 19(4):3083-3095.

http://www.aloki.hu • ISSN 15891623 (Print) • ISSN1785 0037 (Online)

DOI: http://dx.doi.org/10.15666/aeer/1904_30833095

(c) 2021, ALÖKI Kft., Budapest, Hungary 
The PGR treatment, from level 1 up to level 3, significantly increased the K content $(P<0.05)$ compared with the control. $\mathrm{Mg}$ and $\mathrm{S}$ content varied significantly between the tested PGR levels; however, the contents varied inconsistently between the PGRs levels. The lowest $\mathrm{Cu}$ content was measured when PGRs were applied at level 3 compared with the control and other levels.

The $\mathrm{C}, \mathrm{N}, \mathrm{P}$, and $\mathrm{Ca}$ content, as well as the $\mathrm{C}: \mathrm{N}$ ratio, were significantly affected by the simulated hail damage, and differed between the PGR levels due to the interaction between simulated hail damage and PGRs (Table 2). The interaction showed that most leaf mineral content varied between PGR levels on plants that endured some level of simulated hail damage. In plants that endured $100 \%$ simulated hail damage, the mean concentration of $\mathrm{C}$ declined by $11.8 \%$ when PGRs were applied at level 1, compared with the control. $\mathrm{N}$ content increased by $9.6 \%$ when PGRs were applied at level 3 compared with the control; however, where plants suffered $100 \%$ simulated hail damage, the concentration did not significantly differ from levels 1 and 2 . As expected, the $\mathrm{C}: \mathrm{N}$ ratio significantly declined when PGRs were applied at level 1 on plants that endured $100 \%$ simulated hail damage: higher levels of PGRs did not necessarily improve the C:N ratio.

The significant interaction between simulated hail damage and PGR $(P<0.05)$ shows that the application of PGRs (all levels) only increased the P content in $100 \%$ simulated hail-damage plants (Table 2). In 50\% simulated hail-damaged plants, Ca content increased exponentially with the application of PGRs application from level 1, which contributed to the significant interaction between simulated hail damage and PGRs $(P<0.05$; Table 2).

Multivariate data analysis, using PCA, was applied to increase the interpretability of the inconclusive leaf mineral data (Table 3 and Figure 1). The score plot and loading matrix, based on the first and second principal components (PC1 and PC2), accounted for $73.01 \%$ of the total variance.

Table 3. Principal component analysis of the leaf mineral composition of rose geranium plant leaves

\begin{tabular}{c|c|c|c|c}
\hline Principal Component & PC1 & PC 2 & PC 3 & PC4 \\
\hline Eigenvalue & 7.12 & 3.10 & 1.31 & 1.13 \\
Variability (\%) & 50.87 & 22.14 & 9.37 & 8.04 \\
Cumulative (\%) & 50.87 & 73.01 & 82.38 & 90.42 \\
\hline \multicolumn{4}{|c}{ Factor loadings } \\
\hline C & $\mathbf{0 . 6 9}$ & 0.13 & 0.11 & 0.00 \\
N & $\mathbf{0 . 6 8}$ & 0.01 & 0.02 & 0.03 \\
C:N ratio & $\mathbf{0 . 7 8}$ & 0.00 & 0.05 & 0.00 \\
P & $\mathbf{0 . 6 2}$ & 0.01 & 0.27 & 0.04 \\
K & $\mathbf{0 . 7 0}$ & 0.07 & 0.01 & 0.18 \\
Ca & 0.11 & $\mathbf{0 . 6 4}$ & 0.00 & 0.17 \\
$\mathrm{Mg}$ & $\mathbf{0 . 7 7}$ & 0.09 & 0.07 & 0.00 \\
$\mathrm{~S}$ & 0.45 & 0.44 & 0.08 & 0.02 \\
$\mathrm{~B}$ & 0.35 & 0.26 & 0.04 & 0.17 \\
$\mathrm{Cu}$ & 0.21 & 0.24 & 0.08 & 0.41 \\
$\mathrm{Fe}$ & $\mathbf{0 . 5 9}$ & 0.34 & 0.00 & 0.05 \\
$\mathrm{Mn}$ & $\mathbf{0 . 8 2}$ & 0.01 & 0.05 & 0.04 \\
$\mathrm{Zn}$ & 0.36 & 0.20 & 0.33 & 0.02 \\
$\mathrm{Na}$ & 0.00 & $\mathbf{0 . 6 5}$ & 0.20 & 0.01 \\
\hline
\end{tabular}

Values in bold correspond to the factor with the greatest squared cosine 


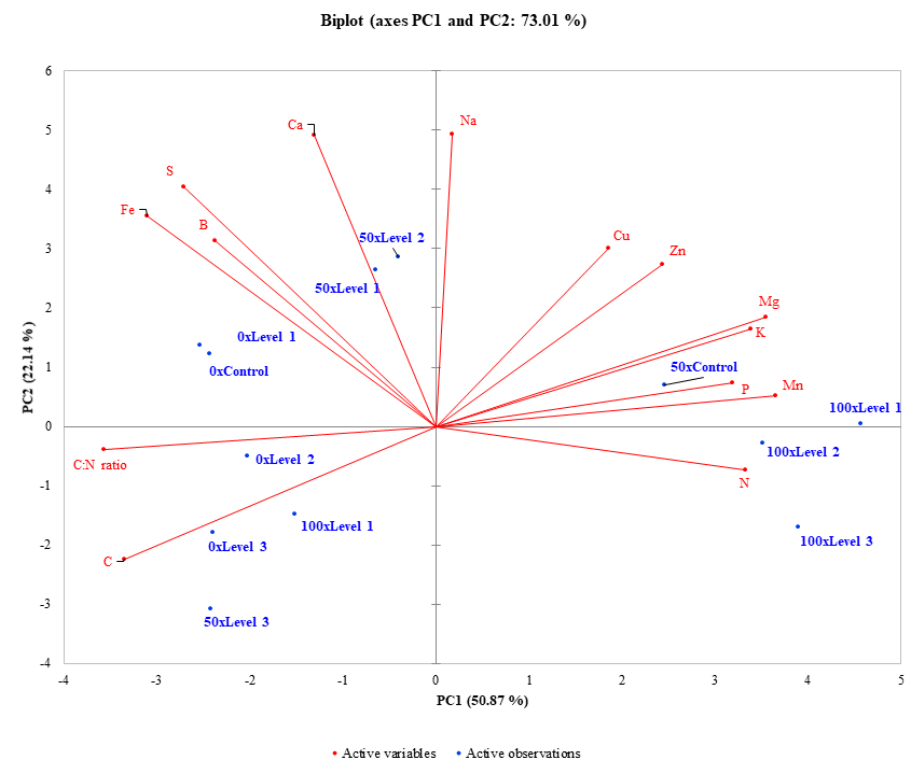

Figure 1. Principal component biplot showing the variations between leaf mineral content ( $C$ $m g / k g, N m g / k g, C: N$ ratio, $P \mathrm{mg} / \mathrm{kg}, \mathrm{K} \mathrm{mg} / \mathrm{kg}, \mathrm{Ca} \mathrm{mg/kg,} \mathrm{Mg} \mathrm{mg/kg,} \mathrm{S} \mathrm{mg/kg,} \mathrm{B} \mathrm{ug/kg,} \mathrm{Cu}$ $u g / \mathrm{kg}, \mathrm{Fe} \mathrm{mg} / \mathrm{kg}, \mathrm{Mn} u \mathrm{~g} / \mathrm{kg}, \mathrm{Zn} u g / \mathrm{kg}$, and $\mathrm{Na} \mathrm{mg} / \mathrm{kg}$ ) in response to hail-simulated damage and the application of PGRs

PC $1(50.87 \%)$ revealed that $\mathrm{N}, \mathrm{P}, \mathrm{K}, \mathrm{Mg}$ and $\mathrm{Mn}$ were positively correlated; however, $\mathrm{Ca}$ and $\mathrm{Na}$ content were the only leaf minerals positively correlated with PGR application and simulated hail damage in PC $2(22.14 \%)$. The PCA confirms that all plants that endured simulated hail damage, at any level, can accumulate leaf minerals when treated with higher levels of PGRs (levels 2 and 3). The $\mathrm{C}$ content and the $\mathrm{C}: \mathrm{N}$ ratio were negatively correlated in PC1, and were lower when PGRs were applied at level 3 and level 1 in the $50 \%$ and $100 \%$ simulated hail damaged plants, respectively.

\section{Leaf phenolic profile}

The chromatogram of the phenolic compounds identified in rose geranium samples using retention indices and the detected masses is presented in Table 4. The analysis of variance showed no statistically significant differences in the phenolic compounds when PGRs were applied, nor were there any interactions between simulated hail damage and PGRs (data not shown). Only the phenolic compounds identified as citric acid, coumaroyl tyrosine, 5-Heptadecylresorcinol, kaempferol-O-hexoside-O-deoxy-hexose and 8.08 (unidentified compound) were significantly affected by the simulated hail damage factor (Figure 2 A-E).

The lowest citric acid content was recorded where plants endured $100 \%$ simulated hail damage $(P<0.05)$, compared with 50\% simulated hail damage and intact plants (Figure $2 A$ ). This indicates that citric acid significantly declines when plants are defoliated. The mean coumaroyl tyrosine content in intact plants was significantly higher $(P<0.05)$ than in the $100 \%$ simulated hail damage plants $(165.28 \mathrm{GAE} / \mathrm{g}$ dry weight vs. 106.49 GAE/g dry weight, respectively; Figure $2 B$ ). With $100 \%$ simulated hail damage, mean concentrations of 5-Heptadecylresorcinol, kaempferol-O-hexoside-O-deoxyhexose and 8.08 (unidentified compound) were significantly higher than in the intact plants (Figure $2 C-E$ ). As hypothesized, the PGRs did not affect these phenolics $(P<0.05)$. 


$$
\text { - } 3090-
$$

Table 4. Phenolic compound identified from the chromatogram

\begin{tabular}{c|c|c|c}
\hline Comp. ID & Compounds name & Quant. Mass $(\boldsymbol{m} / \mathbf{z})$ & RT (Min) \\
\hline 2.11 & Citric acid & 191 & 2.11 \\
8.35 & Protocatechuic acid O-hexoside & 315 & 8.35 \\
9.16 & Isorhamnetin-O-hexoside-O-deoxy-hexose & 179 & 9.16 \\
9.17 & Caffeic acid & 311 & 9.17 \\
9.18 & Caffeoyl tartaric acid & 165 & 9.18 \\
9.60 & Coumaroyl hexose & 299 & 9.60 \\
10.33 & Avenanthramide 2p & 349 & 10.33 \\
12.45 & 5-Heptadecylresorcinol & 327 & 12.45 \\
12.86 & p-Coumaroyl tyrosine & 329 & 12.86 \\
13.50 & Avenanthramide 2f & 431 & 13.53 \\
13.53 & 5-Tricosenylresorcinol & 461 & 13.84 \\
14.59 & 5-Pentacosylresorcinol & 539 & 14.59 \\
15.70 & Oleuropein & 475 & 15.70 \\
16.10 & Chicoric acid & 377 & 16.10 \\
17.12 & 5-Nonadecylresorcinol & 433 & 17.12 \\
18.33 & Ellagic acid arabinoside & 593 & 18.33 \\
18.53 & Kaempferol-O-hexoside-O-deoxy-hexose & 373 & 18.53 \\
18.65 & 5-Nonadecylresorcinol & 525 & 18.65 \\
18.77 & Ligstroside & 527 & 18.77 \\
19.32 & Demethyloleuropein & 419 & 19.32 \\
20.87 & Oleoside dimethylester & & 20.87 \\
\hline
\end{tabular}

Comp. ID = Compound identity; Quant. Mass = quantitative mass
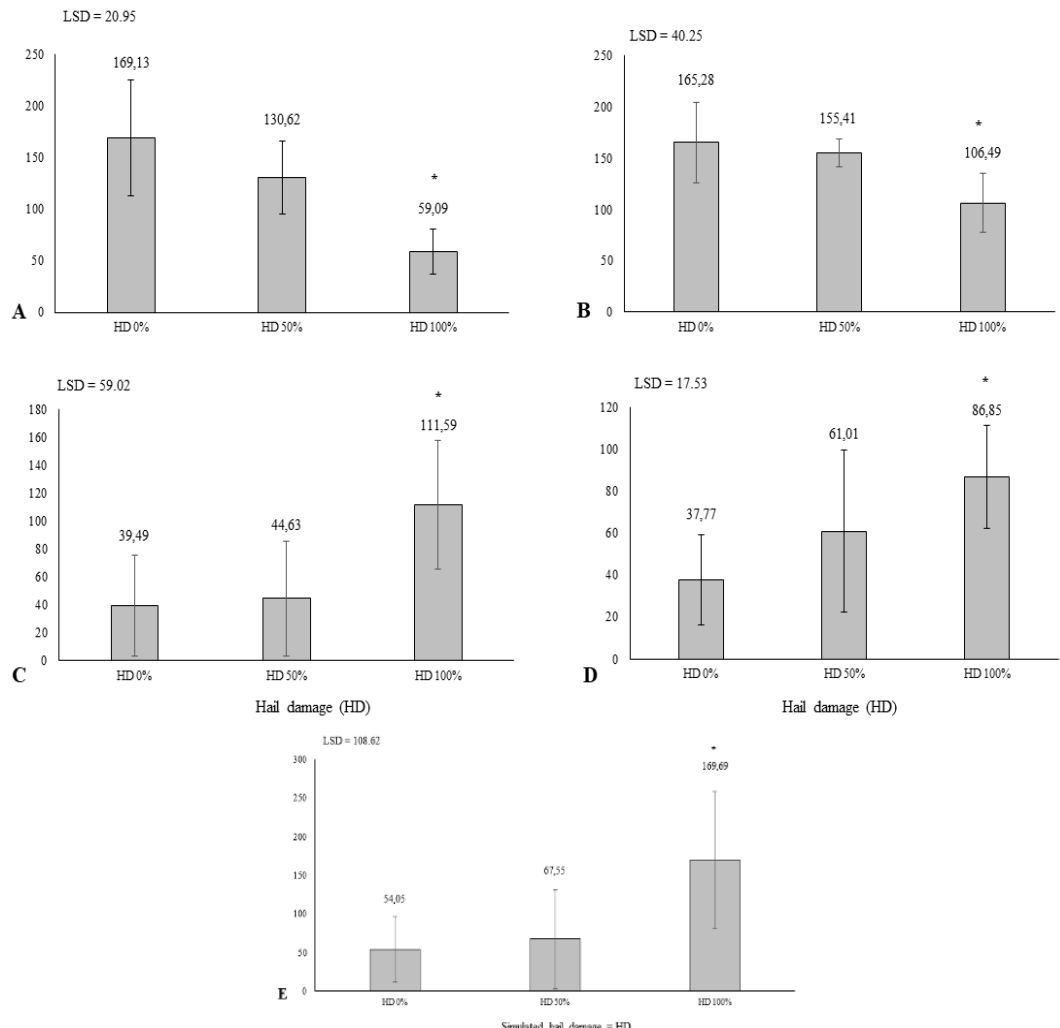

Figure 2. Citric acid A), Coumaroyl tyrosine B), 5-Heptadecylresorcinol C), Kaempferol-Ohexoside-O-deoxy-hexose D) concentration and 8.08 (unidentified compound: E) of simulated hail-damaged rose geranium plants. Standard error (SE) is shown as black bars. Asterisks indicate significant differences 
PCA was used to reduce the redundancy of the leaf phenolics data. Citric acid, coumaroyl hexose, 5-Heptadecylresorcinol, kaempferol-O-hexoside-O-deoxy-hexose and other unidentified compounds were the only phenolics with the largest squared cosine factor (data not shown). The score plot and loading matrix accounted for $41.30 \%$ $(\mathrm{PC} 1=26.01 \%$ and $\mathrm{PC} 2=15.29 \%)$ of the total variance (Figure 3$)$.

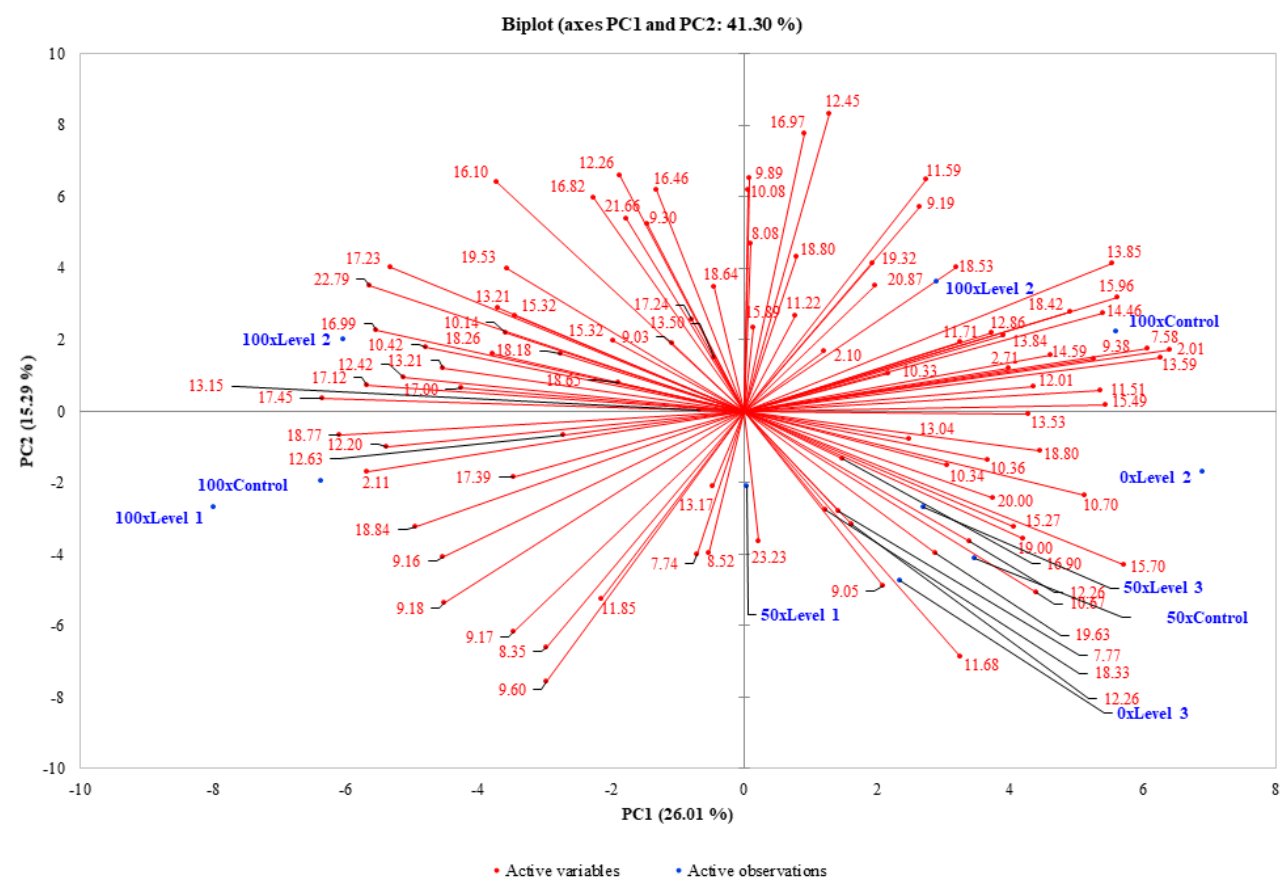

Figure 3. Principal component biplot showing the variation between leaf phenolic compounds. Key: $2.11=$ Citric acid; $8.35=$ Protocatechuic acid O-hexoside; $9.16=$ Isorhamnetin-O hexoside-O-deoxy-hexose; $9.17=$ Caffeic acid; $9.18=$ Caffeoyl tartaric acid; $9.60=$ Coumaroyl hexose $; 10.33=$ Avenanthramide $2 p ; 12.45=5$-Heptadecylresorcinol; $12.86=p$ Coumaroyl tyrosine; $13.50=$ Avenanthramide $2 f ; 13.53=5$-Tricosenylresorcinol; $14.59=5$ Pentacosylresorcinol; $15.70=$ Oleuropein; $16.10=$ Chicoric acid; $17.12=5$ -

Nonadecylresorcinol; $18.33=$ Ellagic acid arabinoside $; 18.53=$ Kaempferol-O-hexoside-O deoxy-hexose; $18.65=5$-Nonadecylresorcinol; $18.77=$ Ligstroside $; 19.32=$

Demethyloleuropein; 20.87 = Oleoside dimethylester (other compounds were unidentified)

The PCA showed a strong positive correlation between 5-Heptadecylresorcinol, kaempferol-O-hexoside-O-deoxy-hexose and other unidentified compounds in PC 1 (Figure 3). The biplot indicates that these phenolic compounds were characterized by the application of distilled water (control) and PGRs at level 2, in 100\% simulated haildamaged plants. PC 1 further showed that citric acid and coumaroyl hexose compounds were negatively correlated in plants that sustained $100 \%$ simulated hail damage, and characterized by the application of distilled water and a low concentration of PGRs (level 1). In PC 2, unidentified compounds $(9.19,11.68,12.42$, and 16.97) were the only compounds with values that correspond for each variable to the factor for which the squared cosine is the largest (Figure 3). 


\section{Discussion}

Plants respond differently to wounding stress following hail damage. Defoliation and wounding stress in plants result in a knock-back effect that reduces the assimilation of $\mathrm{C}$, ultimately affecting the rate of photosynthesis (Kundu and Gantait, 2017). This stress stimulates the production of reactive oxygen species levels, accumulation of the phenolic composition, transient $\mathrm{Ca}^{+2}$ influxes into the cytoplasm, and protein phosphorylation (Bernards and Båstrup-Spohr, 2008; Smékalová et al., 2014). Some plants positively adapt with altered growth habits to contradict the damaging effects of hail, thus, the responses are both reversible and irreversible modifications, such as mineral acquisition, and cell division (Kundu and Gantait, 2017). The activation and regulation of defence mechanism of plants following hail damage and defoliation are primarily driven by upregulation of endogenous phytohormones (Ikeuchi et al., 2017; Nanda and Melnyk, 2018). Nemhauser et al. (2006) and Parađiković et al. (2019) found that exogenous applications of PGRs influence plant stature, organ size, nutrient acquisition, and the antioxidant capacity of plant tissue.

In this study, a majority of the leaf minerals, except $\mathrm{S}, \mathrm{B}, \mathrm{Fe}$ and $\mathrm{Na}$, increased with simulated hail damage, with $100 \%$ simulated hail damage causing statistically significant mineral accumulation. Previous research has shown that defoliated plants exhibit increased leaf elemental concentrations to compensate for the loss of leaf tissue, which is in line with this study (Iqbal et al., 2012). On the other hand, Dada and Oworu (2010) also reported that some plants react to defoliation with a decline of some leaf minerals.

According to Ramage and Williams (2002), mineral nutrients form a significant component of the leaf morphogenesis, depending primarily on the composition and ratio of PGRs. In this study, intact and refoliating plants leaves were affected by the application of PGRs, at any level, which could be attributed to the relationship between the mineral compositions and the exogenously applied PGRs. Kudoyarova et al. (2015) reported that changes in plant mineral availability influences the expression of genes controlling hormones, metabolism and signaling. Therefore, the application of PGRs may directly improve the acquisition of minerals associated with growth and development plants (Pankoke and Müller, 2013). This correlates with the findings of a number of previous studies, for example, Oliveira et al. (2010) reported that CK stimulate the accumulation of $\mathrm{Mg}$ in pond apple (Annona glabra L.), and Vinković et al. (2012) reported that tomatoes (Lycopersicon esculentum Mill.) treated with biostimulants containing PGRs increased the $\mathrm{K}$ content, compared to control plants. In an in vitro study, $\mathrm{P}$ was rapidly consumed in shoot formation, corresponding to the initiation and growth of the shoots (Ramage and Williams, 2002). Accumulated Ca content in refoliating leaves could be ascribed to the interaction between $\mathrm{Ca}$ and $\mathrm{CK}$ signal transduction, leading to cell division (Saunders, 1992). PCA confirmed that N, P, K, Ca, Mg, Mn and Na, minerals mostly associated with plant growth were positively correlated where plants endured simulated hail damage and treated with PGRs at levels 2 and 3.

Smékalová et al. (2014) reported that defoliation reduce the assimilation of $\mathrm{C}$, and the $\mathrm{N}$ acquisition, directly affecting the $\mathrm{C}: \mathrm{N}$ ratio of the plant. In this study, low $\mathrm{C}$ content resulting from the application of PGRs at level 1 caused the declined $\mathrm{C}: \mathrm{N}$ ratio where plants endured $100 \%$ simulated hail damage. Bryant et al. (1993) reported that the C:N ratio declines following severe defoliation, caused by nutritional stress resulting from severe defoliation. In addition, the concentration of phytohormones with inhibitory effects, increases following severe defoliation and wounding. This increase suppresses the stimulatory phytohormones associated with mineral acquisition and photosynthetic 
activities (Ikeuchi et al., 2017; Nanda and Melnyk, 2018; Pommerrenig et al., 2019). Therefore, a low $\mathrm{C}: \mathrm{N}$ ratio in plants treated with level 1 PGRs could be due to the antagonistic crosstalk between the phytohormones with inhibitory effects, such as abscisic acid, and growth stimulatory phytohormones, such as GA, BR and CK (Pommerrenig et al., 2019). The PCA also confirms that the C:N ratio is reduced when plants endure hail damage.

Specific phenolic compounds respond differently to defoliation (Roitto et al., 2003). The different branches of phenolic compounds' biosynthetic pathway may compete for substrates, and these internal metabolic trade-offs may result in the differential accumulation of phenolic compounds, such as citric acid and coumaroyl tyrosine, observed in this study, particularly during refoliation (Roitto et al., 2003). The accumulation of these phenolic compounds are a typical plant response to defoliation, including wounding stress (Bryant et al., 1993; Nanda and Melnyk, 2018). According to Walters et al. (2003), the accumulation of these phenolic compounds also act as defense compounds involved in lignification and modification of the plant cell walls. The PCA confirms that $100 \%$ hail-damaged rose geranium plants accumulate most of the phenolic compounds associated with defoliation and wounding, while those with only $50 \%$ defoliation accumulate similar phenolic compounds as $0 \%$ defoliated plants. Furthermore, the application of PGRs does not necessarily affect these phenolics.

\section{Conclusion}

The results indicate that hail-damaged rose geranium plants will accumulate leaf minerals when treated with higher levels of PGRs (level 2; GA-2.55 mg/kg, BR-1.02 mg/kg, and traces of CKs-0.05 mg/kg, and level 3; GA-3.83 mg/kg, BR-1.53 mg/kg, and CKs $-0.075 \mathrm{mg} / \mathrm{kg}$ ). Post-hail damage recovery strategies using PGRs may be an alternative strategy for improving the leaf mineral status, assisting the plants to recover lost herbage material. Completely hail-damaged rose geranium plants (100\% defoliation) will most likely accumulate citric acid, coumaroyl hexose, 5-Heptadecylresorcinol, kaempferol-O-hexoside-O-deoxy-hexose, while those with only $50 \%$ defoliation accumulate similar phenolic compounds as intact plants. This study contributed to the development of a mitigation strategy for hail-damaged rose geranium plants using agricultural PGRs. Practically, it was also recorded (data not provided) that the herbage yield and essential oil quality improves when the PGR-mixture is applied at level 3 (GA-3.83 mg/kg, BR-1.53 mg/kg, and CKs- $0.075 \mathrm{mg} / \mathrm{kg}$ ) on rose geranium plants with more than $50 \%$ foliage loss. It may be beneficial to test other sources of biostimulants as an alternative strategy for future studies.

Acknowledgements. Authors would like to thank Agraforum (Germany) for providing the PGRs (registered as Lucky Plant ${ }^{\circledR}$ : EP1051075B1 \& EP1933626), and the National Research Foundation (Thuthuka Funding Instrument: PhD Track, and Black Academics Advancement Programme) for financial assistance.

\section{REFERENCES}

[1] Bal, S. K., Saha, S., Fand, B. B., Singh, N. P., Rane, J., Minhas, P. S. (2014): Hailstorms: Causes, damage and post-hail management in agriculture. - National Institute of Abiotic Stress Management Technical Bulletin-5: India. 
[2] Bernards, M. A., Båstrup-Spohr, L. (2008): Phenylpropanoid metabolism induced by wounding and insect herbivory. - Springer: Dordrecht.

[3] Bryant, J. P., Reichardt, P. B., Clausen, T. P., Werner, R. A. (1993): Effects of mineral nutrition on delayed inducible resistance in Alaska paper birch. - Ecology 74: 2072-2084.

[4] Changnon, S. A. (1999): Data and approaches for determining hail risk in the contiguous United States. - Journal of Applied Meteorology 38: 1730-1739.

[5] Combrink, N. J. J. (2005): Nutrient solutions and greenhouse management. - Combrink Family Trust: Stellenbosch.

[6] Dada, O. A., Oworu, O. O. (2010): Mineral and nutrient leaf composition of two cassava (Manihot esculenta Crantz) cultivars defoliated at varying phenological phases. - Notulae Scientia Biologicae 5: 44-48.

[7] Dhaliwal, G. S., Gupta, N., Kukal, S. S., Kaur, M. (2014): Standardization of automated vario EL III CHNS analyzer for total carbon and nitrogen determination in plants. Communications in Soil Science and Plant Analysis 45: 1316-1324.

[8] Ikeuchi, M., Iwase, A., Rymen, B., Lambolez, A., Kojima, M., Takebayashi, Y., Heyman, J., Watanabe, S., Seo, M., De Veylder, L., Sakakibara, H., Sugimoto, K. (2017): Wounding triggers callus formation via dynamic hormonal and transcriptional changes. - Plant Physiology 175: 1158-1174.

[9] Iqbal, S., Younas, U., Chan, K. W., Zia-Ul-Haq, M., Ismail, M. (2012): Chemical composition of Artemisia annua L. leaves and antioxidant potential of extracts as a function of extraction solvents. - Molecules 1: 6020-6032.

[10] Irigoyen, I., Domeno, I., Muro, J. (2010): Effect of defoliation by simulated hail damage on yield of potato cultivars with different maturity performed in Spain. - American Journal of Potato Research 88: 82-90.

[11] Kim, M. Y., Lee, B. W., Lee, H. U., Lee, Y. Y., Kim, M. H., Lee, J. Y., Lee, B. K., Woo, K. S., Kim, H. J. (2019): Phenolic compounds and antioxidant activity in sweet potato after heat treatment. - Journal of the Science of Food and Agriculture 99: 6833-6840.

[12] Kudoyarova, G. R., Dodd, I. C., Veselov, D. S., Rothwell, S. A., Veselov, S. Y. (2015): Common and specific responses to availability of mineral nutrients and water. - Journal of Experimental Botany 66(8): 2133-2144.

[13] Kundu, S., Gantait, S. (2017): Abscisic acid signal crosstalk during abiotic stress response. - Plant Gene 11: 61-69.

[14] Maia, J. G. S., Andrade, E. H. A., Couto, H. A., da Silva, A. C. M., Marx, F., Henke, C. (2007): Plant sources of Amazon rosewood oil. - Quimica Nova Chemistry in Brazil 30: 1906-1910.

[15] Masson, P., Alves, A. C., Ebbels, T. M., Nicholson, J. K., Want, E. J. (2010): Optimization and evaluation of metabolite extraction protocols for untargeted metabolic profiling of liver samples by UPLC-MS. - Analytical Chemistry 82: 7779-7786.

[16] Mishra, S., Kumar, A., Yadav, S., Singhal, M. K. (2018): Assessment of heavy metal contamination in water of Kali River using principle component and cluster analysis, India. - Sustainable Water Resources Management 4: 573-581.

[17] Nanda, A. K., Melnyk, C. W. (2018): The role of plant hormone during grafting. - Semiin-vivo Developmental Biology 131: 49-58.

[18] Nemhauser, J. L., Hong, F. X., Chory, J. (2006): Different plant hormones regulate similar processes through largely non-overlapping transcriptional responses. - Cell 126: 467-475.

[19] Nielsen, R. L. B. (2012): Recovery from hail damage to young corn. - Corny News Network, June 2015.

[20] Obeso, J. R. (2002): The costs of reproduction in plants. - New Phytologist 155: 321-348.

[21] Oliveira, L. M., Paiva, R., Santana, J. R., Pereira, F. D., Nogueira, R. C., Silva, L. C. (2010): Effects of cytokinins on in vitro mineral accumulation and bud development in Annona glabra L. - Ciência e Agrotecnologia 34: 1439-1445. 
[22] Pankoke, H., Müller, C. (2013): Impact of defoliation on the regrowth capacity and the shoot metabolite profile of Plantago lanceolata L. - Plant Physiology and Biochemistry 71: 325-333.

[23] Parađiković, N., Teklić, T., Zeljković, S., Lisjak, M., Špoljarević, M. (2019): Biostimulants research in some horticultural plant species-A review. - Food and Energy Security 8: e00162.

[24] Pommerrenig, B., Eggert, K., Bienert, G. P. (2019): Boron deficiency effects on sugar, ionome, and phytohormone profiles of vascular and non-vascular leaf tissues of common plantain (Plantago major L.). - International Journal of Molecular Sciences 20: 3882.

[25] Ramage, C. M., Williams, R. R. (2002): Mineral nutrition and plant morphogenesis. - In Vitro Cellular \& Developmental Biology-Plant 38: 116-124.

[26] Roitto, M., Markkola, A., Julkunen-Tiitto, R., Sarjala, T., Rautio, P., Kuikka, K., Tuomi, J. (2003): Defoliation-induced responses in peroxidases, phenolics, and polyamines in Scots pine (Pinus sylvestris L.) needles. - Journal of Chemical Ecology 29: 1905-1918.

[27] Saunders, M. J. (1992): Cytokinin signal transduction through $\mathrm{Ca}^{2+}$ in mosses. - In progress in plant growth regulation, Springer: Dordrecht.

[28] Sedibe, M. M., Allemann, J. (2012): Yield and quality response of rose geranium (Pelargonium graveolens L.) to sulphur and phosphorus application. - South African Journal of Plant and Soil 29: 151-156.

[29] Shapiro, S. S., Wilk, M. B. (1965): An analysis of variance test for normality (complete samples). - Biometrika 52: 591-611.

[30] Smékalová, V., Doskočilova, A., Komis, G., Šamaj, J. (2014): Crosstalk between secondary messengers, hormones and MAPK modules during osmotic stress signaling in plants. - Biotechnology Advances 32: 2-11.

[31] Stander, M. A., Van Wyk, B. E., Taylor, M. J., Long, H. S. (2017): Analysis of phenolic compounds in rooibos tea (Aspalathus linearis) with a comparison of flavonoid-based compounds in natural populations of plants from different regions. - Journal of Agricultural and Food Chemistry 65: 10270-10281.

[32] Steel, R. G. D., Tourie, J. H. (1980): Principles and procedures of statistics: biometrical approach (eds.). - McGraw-Hill, Kogakusha, Tokyo: Japan.

[33] Treutter, D. (2010): Managing phenol contents in crop plants by phytochemical farming and breeding-Visions and constraints. - International Journal of Molecular Sciences 11: 807-857.

[34] Vinković, T., Parađiković, N., Tkalec, M., Teklić, T., Lončarić, Z. (2012): Effect of biostimulants on nutrient content in some organs and tomato plant. - In: Pospišil, M. (ed.) Book of abstract of 47th Croatian and 7th International Symposium on Agriculture. 2012 February 13-17, Faculty of agronomy, University of Zagreb.

[35] Walters, D. (2003): Resistance to plant pathogens: possible roles for free polyamines and polyamine catabolism. - New Phytologist 159: 109-115.

[36] Wani, S. H., Kumar, V., Shriram, V., Kumar Sah, S. (2016): Phytohormones and their metabolic engineering for abiotic stress tolerance in crop plants. - The Crop Journal 4(3): $162-176$. 